\title{
A NOTE ON THE INTERPOLATION OF THE DIFFIE-HELLMAN MAPPING
}

\author{
ARNE WINTERHOF
}

\begin{abstract}
We obtain lower bounds on the degrees of polynomials representing the Diffie-Hellman mapping $f\left(\gamma^{x}, \gamma^{y}\right)=\gamma^{x y}$, where $\gamma$ is a nonzero element of $F_{q}$ of order $d, x$ runs through a subset of $[0, d-1]$, and $y$ runs through a set of consecutive integers.
\end{abstract}

\section{INTRODUCTION}

Let $q$ be a prime power, $F_{q}$ be the finite field of order $q$, and $\gamma$ be a nonzero element in $F_{q}$ of order $d \mid q-1$. The Diffie-Hellman problem in $F_{q}$ is the following. Let $\gamma^{x}, \gamma^{y}$ be elements of $F_{q}$. Find $\gamma^{x y}$ without knowing $x$ and $y$. The Diffie-Hellman key exchange (see for example [4]) is based on the fact that no easy representation of the Diffie-Hellman mapping

$$
f\left(\gamma^{x}, \gamma^{y}\right)=\gamma^{x y} \quad \text { for } 0 \leqslant x, y<d
$$

is known. It can easily be verified that the polynomial

$$
f(X, Y)=d^{-1} \sum_{i, j=0}^{d-1} \gamma^{-i j} X^{i} Y^{j}
$$

satisfies (1), where $d^{-1}$ denotes the inverse of $d$ modulo the characteristic of $F_{q}$. Under the natural restriction that $\operatorname{deg}_{X}(f), \operatorname{deg}_{Y}(f)<d$ this polynomial is uniquely determined. It has the largest possible degree $2(d-1)$ and the largest number of nonzero coefficients $d^{2}$. For breaking the Diffie-Hellman cryptosystem it would be sufficient to have an easy polynomial satisfying $f\left(\gamma^{x}, \gamma^{y}\right)=\gamma^{x y}$ for all pairs $(x, y) \in \mathcal{W}$ of a large subset $\mathcal{W} \subseteq$ $[0, d-1]^{2}$.

Recently, El Mahassni and Shparlinski [2] obtained the following result for $d=q-1$ extending the technic in [1]. Let $\mathcal{W} \subseteq[N+1, N+H]^{2}$ with $2 \leqslant H \leqslant q-1$ and let $f(X, Y) \in F_{q}[X, Y]$ be a polynomial such that

$$
f\left(\gamma^{x}, \gamma^{y}\right)=\gamma^{x y} \quad \text { for all }(x, y) \in \mathcal{W}
$$

If $|\mathcal{W}| \geqslant 10 H^{8 / 5}$ then we have

$$
\operatorname{deg}(f) \geqslant \frac{|\mathcal{W}|^{2}}{128 H^{3}}
$$

Received 9th April, 2001

Copyright Clearance Centre, Inc. Serial-fee code: 0004-9727/01 \$A2.00+0.00. 
In this note we prove a lower bound on $\operatorname{deg}(f)$ for some different $\mathcal{W}$ using direct interpolation.

THEOREM 1. Let $q$ be a prime power, $\gamma$ be a nonzero element of $F_{q}$ of order $d \mid q-1$, and $N$ be an integer. Let $\mathcal{U}$ be a set of distinct integers modulo $d$, and $\mathcal{V} \subseteq\{N+1, \ldots, N+H\}$ with $|\mathcal{V}|=H-s$ and $1 \leqslant H<d$. Let $f(X, Y) \in F_{q}[X, Y]$ be a polynomial satisfying

$$
f\left(\gamma^{x}, \gamma^{y}\right)=\gamma^{x y} \quad \text { for all }(x, y) \in \mathcal{U} \times \mathcal{V} .
$$

Then we have the following lower bound on the total degree of $f(X, Y)$ :

$$
\operatorname{deg}(f) \geqslant \min \left(|\mathcal{U}|,\left\lceil\frac{H-s}{s+1}\right]\right)-1
$$

For $d=q-1$ this result and the result in [2] complement each other. In particular, Theorem 1 contains nontrivial results for certain subsets $\mathcal{W}$ of cardinality smaller than $10 H^{8 / 5}$. In contrast to the method in the present note the method in [2] looses its power for $d<q-1$.

\section{Proof of the Theorem}

Put

$$
n=\min (|\mathcal{U}|,[(H-s) /(s+1)\rceil)-1
$$

Obviously, $\mathcal{V}$ contains a subset $\left\{v_{0}, \ldots, v_{n}\right\}$ of consecutive integers. Then we have

$$
f\left(\gamma^{u_{i}}, \gamma^{v_{j}}\right)=\gamma^{u_{i} v_{j}} \quad \text { for } 0 \leqslant i, j \leqslant n,
$$

where $u_{0}, \ldots, u_{n}$ are distinct elements of $\mathcal{U}$. Since otherwise the result is trivial we may suppose that $\operatorname{deg}_{X}(f), \operatorname{deg}_{Y}(f) \leqslant n$, that is

$$
f(X, Y)=\sum_{i, j=0}^{n} c_{i, j} X^{i} Y^{j}
$$

The coefficients $c_{i j}$ are uniquely determined by the following matrix equation,

$$
\begin{aligned}
C & =\left(\begin{array}{ccc}
c_{0,0} & \cdots & c_{0, n} \\
\vdots & & \vdots \\
c_{n, 0} & \cdots & c_{n, n}
\end{array}\right) \\
& =\left(\begin{array}{cccc}
1 & \gamma^{u_{0}} & \cdots & \gamma^{u_{0} n} \\
\vdots & \vdots & & \vdots \\
1 & \gamma^{u_{n}} & \cdots & \gamma^{u_{n} n}
\end{array}\right)^{-1}\left(\begin{array}{ccc}
\gamma^{u_{0} v_{0}} & \cdots & \gamma^{u_{0} v_{n}} \\
\vdots & & \vdots \\
\gamma^{u_{n} v_{0}} & \cdots & \gamma^{u_{n} v_{n}}
\end{array}\right)\left(\begin{array}{ccc}
1 & \cdots & 1 \\
\gamma^{v_{0}} & \cdots & \gamma^{v_{n}} \\
\vdots & & \vdots \\
\gamma^{v_{0} n} & \cdots & \gamma^{v_{n} n}
\end{array}\right)^{-1} .
\end{aligned}
$$


On the right hand side we have a product of regular matrices and hence $C$ itself is regular. In particular, there exist some nonzero elements in every row of $C$ which yields the result.

REMARKS. 1. In [2] results on the degree of polynomials $F(X, Y, Z)$ over $F_{q}$ satisfying $F\left(\gamma^{x}, \gamma^{y}, \gamma^{x y}\right)=0$ are also obtained, where $(x, y)$ runs through a certain subset of $[1, q-$ $1]^{2}$. The direct interpolation does not work for these polynomials.

2. For univariate polynomials $h(X) \in F_{q}[X]$ satisfying $h\left(\gamma^{x}\right)=\gamma^{x^{2}}$ for $x$ in a certain subset of $[0, q-2]$, which are closely related to the Diffie-Hellman mapping, similar results are obtained in [1] and [5, Section 8]. For the unique polynomial $h(X)$ of degree at most $q-2$ defined in the whole interval $[0, q-2]$ an exact formula is given in [3].

\section{REFERENCES}

[1] D. Coppersmith and I. Shparlinski, 'On polynomial approximation of the discrete logarithm and the Diffie-Hellman mapping', J. Cryptology 13 (2000), 339-360.

[2] E. El Mahassni and I. Shparlinski, 'Polynomial representations of the Diffie-Hellman mapping', Bull. Austral. Math. Soc. 63 (2001), 467-473.

[3] W. Meidl and A. Winterhof, 'A polynomial representation of the Diffie-Hellman mapping', (preprint).

[4] A.J. Menezes, P.C. van Oorschot and S.A. Vanstone, Handbook of applied cryptography (CRC Press, Boca Raton, 1997).

[5] I.E. Shparlinski, Number theoretic methods in cryptography (Birkhäuser, Basel, 1999).

Institute of Discrete Mathematics

Austrian Academy of Sciences

Sonnenfelsgasse $19 / 2$

A-1010 Vienna

Austria

e-mail: arne.winterhof@oeaw.ac.at 